LANDAU-94-TMP-8

submited to Mod. Phys. Lett. A

Dec 1994

\title{
GEOMETRICAL DESCRIPTION OF THE LOCAL INTEGRALS OF MOTION OF MAXWELL-BLOCH EQUATION
}

\author{
A. V. Antonov, ${ }^{*}$ \\ Landau Institute for Theoretical Physics, \\ Kosygina 2, GSP-1, 117940 Moscow V-334, Russia \\ A. A. Belov, ${ }^{* * 2}$ \\ International Institute for Earthquake Prediction Theory \\ and Mathematical Geophysics, \\ Warshavskoe sh., 79, k. 2, Moscow 113556, Russia \\ B. L. Feigin,* \\ Landau Institute for Theoretical Physics, \\ Kosygina 2, GSP-1, 117940 Moscow V-334, Russia
}

We represent a classical Maxwell-Bloch equation and related to it positive part of the AKNS hierarchy in geometrical terms. The Maxwell-Bloch evolution is given by an infinitesimal action of a nilpotent subalgebra $\mathbf{n}_{+}$of affine Lie algebra $\widehat{s l}_{2}$ on a Maxwell-Bloch phase space treated as a homogeneous space of $\mathbf{n}_{+}$. A space of local integrals of motion is described using cohomology methods. We show that hamiltonian flows associated to the Maxwell-Bloch local integrals of motion (i.e. positive AKNS flows) are identified with an infinitesimal action of an abelian subalgebra of the nilpotent subalgebra $\mathbf{n}_{+}$on a Maxwell- Bloch phase space. Possibilities of quantization and latticization of Maxwell-Bloch equation are discussed.

* Supported in part by International Science Foundation (Grant M6N000)

1 E-mail: antonov@landau.ac.ru

** Supported in part by International Science Foundation (Grant N89000)

2 E-mail: mitpan00@iki3.bitnet, subject: belov or mitpan@oka.mitp.free.net, subject: belov 


\section{Introduction}

A powerful cohomological technique was proposed by B. Feigin and E. Frenkel $[1,2]$ for description of a space of local integrals of motion for Toda field theories associated to affine Lie algebra $\mathbf{g}[3,4]$. It was proved that the phase space of the classical Toda theories was isomorphic to the quotient $N_{+} / A_{+}$, where $N_{+}$ was the Lie group of a nilpotent Lie subalgebra $\mathbf{n}_{+}$of algebra $\mathbf{g}$, and $A_{+}$is its principle commutative subgroup. This identification gave possibility to interprete the evolution of the Toda system on its phase space in simple terms. Namely, Toda Hamiltonian action on the phase space coincided with the left infinitesimal action on the nilpotent subalgebra $\mathbf{n}_{+}$on $N_{+} / A_{+}$, and action of Toda local integrals of motion was given by the right infinitesimal action of a commutative subalgebra $\mathbf{a}_{-}$, opposite to the principal commutative subalgera $\mathbf{a}_{+}$with respect to the Cartan involution. The space of local integrals of motion of Toda theory (quantum and classical) was ungerstood as a cohomology class of certain complex. We transfer this technique to the simpliest case of classical Maxwell-Bloch (MB) equation $[3,5,6]$.

Here we will use the following simple form of classical MB equation

$$
\begin{aligned}
& \partial_{\tau} \beta=e^{-\int^{z} d t \beta(t) \gamma(t)}, \\
& \partial_{\tau} \gamma=e^{\int^{z} d t \beta(t) \gamma(t)},
\end{aligned}
$$

where $\beta(z), \gamma(z)$ are functions on the circle $|z|=1, z \in \mathbf{C}$. MB eq. can be rewritten in the zero-curvature form (compare to [5])

$$
\begin{aligned}
B_{1} & =\lambda \sigma_{3}+\left(\begin{array}{cc}
0 & -q \\
r & 0
\end{array}\right), \\
C_{1} & =-\frac{1}{4} \lambda^{-1} e^{-\int^{z} d t \beta(t) \gamma(t)}\left(\begin{array}{cc}
\gamma & -\gamma^{2} \\
1 & -\gamma
\end{array}\right), \\
\partial_{\tau} B_{1} & -\partial C_{1}-\left[B_{1}, C_{1}\right]=0,
\end{aligned}
$$

where $\partial=\partial / \partial z, \sigma_{3}$ is Pauli matrix and $r, q$ are functions $r=\frac{1}{2} \beta, q=\frac{1}{2}\left(-\beta \gamma^{2}+\right.$ $2 \partial \gamma)$.

MB eq. belongs to the AKNS hierarchy [3]

$$
B_{n}(\lambda)=\sum_{j=0}^{n} b_{j} \lambda^{n-j}, \quad C_{n}(\lambda)=\sum_{j=0}^{n-1} c_{j} \lambda^{j-n}, \quad n=1,2, \cdots
$$

where $b_{0}=\sigma_{3} ; b_{j}, c_{j}$ are matrixes $2 \times 2$.

$$
\begin{aligned}
& \partial_{x_{n}} B_{m}-\partial_{x_{m}} B_{n}+\left[B_{m}, B_{n}\right]=0, \\
& \partial_{y_{n}} C_{m}-\partial_{y_{m}} C_{n}+\left[C_{m}, C_{n}\right]=0, \\
& \partial_{y_{n}} B_{m}-\partial_{x_{m}} C_{n}+\left[B_{m}, C_{n}\right]=0,
\end{aligned}
$$


for $m, n=1,2, \cdots$. In MB eq. $\partial_{x_{1}} \equiv \partial, \partial_{y_{1}} \equiv \partial_{\tau}$. We call the first line of this system as positive (with respect to the spectral parameter $\lambda$ ) part of the AKNS hierarchy.

MB eq. is the Hamilton equation

$$
\partial_{\tau} \beta=\{\beta, H\}, \quad \partial_{\tau} \gamma=\{\gamma, H\}
$$

with hamiltonian

$$
\oint d z \beta(z) e^{\int^{z} d t \beta(t) \gamma(t)}-\oint d z \gamma(z) e^{-\int^{z} d t \beta(t) \gamma(t)}
$$

and Poisson brackets

$$
\begin{aligned}
& \{\gamma(x), \beta(y)\}=\delta(x-y)+\frac{1}{2} \epsilon(x-y) \gamma(x) \beta(y), \\
& \{\gamma(x), \gamma(y)\}=-\frac{1}{2} \epsilon(x-y) \gamma(x) \gamma(y), \\
& \{\beta(x), \beta(y)\}=-\frac{1}{2} \epsilon(x-y) \beta(x) \beta(y),
\end{aligned}
$$

where $\epsilon$ is sign function. In this paper we investigate local integrals of motion of $\mathrm{MB}$ eq., i.e. local functionals of the form $\int d z P(\beta(z), \gamma(z))$, where $P$ is a differential polinomial in $\beta, \gamma$. It is known that local integrals of motion of MB eq. coincide with the hamiltonians of the positive part of the AKNS hierarchy.

Consider affine algebra $\widehat{s l}_{2}$ without central extension as defined over formal Laurent power series $\mathbf{C}((t)): \widehat{s l}_{2}=s l_{2} \otimes \mathbf{C}((t)) \oplus \mathbf{C} d, d$ is standart Z-grading operator, $d=t d / d t$. Let $e, h, f$ be standart $s l_{2}$ generators; then define Cartan subalgebra $\mathbf{h}$ of the affine algebra $\widehat{s l}_{2}: \mathbf{h}=\mathbf{C} h \otimes 1 \oplus \mathbf{C} d$. Nilpotent subalgebras $\mathbf{n}_{+}\left(\mathbf{n}_{-}\right)$of $\widehat{s l}_{2}$ are generated by $e, f \otimes t$ (resp. $\left.f, e \otimes t^{-1}\right)$. For $\widehat{s l}_{2}$ we have Cartan decomposition $\widehat{s l}_{2}=\mathbf{n}_{+} \oplus \mathbf{h} \oplus \mathbf{n}_{-}$. Denote $\mathbf{h}_{ \pm}$as commuting subalgebra of $\mathbf{n}_{ \pm}$, generated by $h_{ \pm i}=h \otimes t^{ \pm i}, \quad i=1,2, \cdots$. Let $N_{+}$be the Lie group of the nilpotent subalgebra $\mathbf{n}_{+}$and $H_{+}$be the Lie group of the commutative subalgebra $\mathbf{h}_{+}$.

We will show that the MB phase space is isomorphic to the quotient $N_{+} / H_{+}$.

The action of two parts of MB hamiltonian on the phase space is identified with the left infinitesimal action of the nilpotent subalgebra $\mathbf{n}_{+}$on the quotient $N_{+} / H_{+}$. Then we interpret the space of local integrals of motion of MB eq. as the first cohomology of $\mathbf{n}_{+}$with coefficients in $\mathbf{C}\left(N_{+} / H_{+}\right)$. This formulation allows to describe the space of the MB local integrals of motion and gives opportunity to quantize them [1]. Moreover the action of local integrals of motion (i.e. positive AKNS hamiltonians) on the phase space can be realized as the right infinitesimal action of the commutative subalgebra $\mathbf{h}_{-}$on the quotient $N_{+} / H_{+}$.

This paper is organized as follows. In Section 2 we introduce the MB phase space as reduced bosonic $\beta, \gamma, \phi$ system. The MB hamiltonian is written. We show that an action of the hamiltonian gives rise to an action of nilpotent subalgebra 
$\mathbf{n}_{+}$of $\widehat{s l}_{2}$ on the phase space. In Section 3 geometrical picture of MB eq. is given. We prove that there exists the $\mathbf{n}_{+}$-isomorphism between $M B$ phase space and quoutient $N_{+} / H_{+}$. In Section 4 we give cohomological description of the space of local integrals of motion. In Section 5 we show that vector fields generated by the local integrals of motion of $\mathrm{MB}$ eq. are given by the right infinitesimal action of h_ on $N_{+} / H_{+}$.

\section{Definition and Hamiltonian Picture}

In this section we give the MB eq. as a reduction of the $\beta, \gamma, \phi$ system. Consider three classical bosonic fields $\beta(z), \gamma(z), \partial \phi(z)$ on circle $z \in \mathbf{C},|z|=1$ with Poisson brackets

$$
\begin{aligned}
& \{\gamma(z), \beta(w)\}=\delta(z-w), \\
& \{\phi(z), \phi(w)\}=\frac{1}{2} \epsilon(z-w) .
\end{aligned}
$$

Let $\Pi_{0}$ be space of differential polinomials in $\beta, \gamma, \partial \phi$, i.e. polinomials of the form

$$
P\left(\beta(z), \gamma(z), \partial \phi(z), \partial \beta(z), \partial \gamma(z), \partial^{2} \phi(z), \cdots\right),
$$

$(\partial=\partial / \partial z)$ We will refer to

$$
F[\beta, \gamma, \phi]=\oint d z P\left(\beta(z), \gamma(z), \partial \phi(z), \partial \beta(z), \partial \gamma(z), \partial^{2} \phi(z), \cdots\right)
$$

as local functionals. Denote space of local functionals as $\widehat{\Pi}_{0}$. The space $\widehat{\Pi}_{0}$ can be identified with $\Pi_{0} / \partial \Pi_{0}$, because integral of total derivative is zero. We can ever consider space of functions of the form $P(\beta, \gamma, \partial \phi, \cdots) e^{n \phi}$, where $P \in \Pi_{0}$, which can be treated as $\Pi_{n}=\Pi_{0} \otimes e^{n \phi}$. Denote $\partial^{\prime}=\partial+n \partial \phi$. It is obvious that $\partial\left(P e^{n \phi}\right)=\left(\partial^{\prime} P\right) e^{n \phi}$, so the action of $\partial$ on $\Pi_{n}$ is $(\partial+n \partial \phi) \otimes 1$. Define $\widehat{\Pi}_{n}$ as integrals of elements from $\Pi_{n}$, i.e. $\widehat{\Pi}_{n}=\Pi_{n} / \partial \Pi_{n}$.

Introduce a hamiltonian

$$
H=\oint d z \beta(z) e^{\phi(z)}-\oint d z \gamma(z) e^{-\phi(z)} \in \widehat{\Pi}_{1} \oplus \widehat{\Pi}_{-1}
$$

Consider the Hamilton equation with Poisson brackets (1) and hamiltonian (2)

$$
\begin{aligned}
\partial_{\tau} \beta & =\{\beta, H\}=e^{-\phi}, \\
\partial_{\tau} \gamma & =\{\gamma, H\}=e^{\phi}, \\
\partial_{\tau} \partial \phi & =\{\partial \phi, H\}=\beta e^{\phi}+\gamma e^{-\phi},
\end{aligned}
$$

where $\partial_{\tau}$ designates the derivative over the time $\tau$. 
Define the action of operators $\bar{Q}_{1}, \bar{Q}_{0}$ on the space $\Pi_{0}$

$$
\begin{aligned}
\bar{Q}_{1}: \Pi_{0} \longrightarrow \Pi_{1}, \bar{Q}_{1} & =\left\{\cdots, \oint d z \beta e^{\phi}\right\}, \\
\bar{Q}_{0}: \Pi_{0} \longrightarrow \Pi_{-1}, \bar{Q}_{0} & =\left\{\cdots, \oint d z \gamma e^{-\phi}\right\} .
\end{aligned}
$$

It is easy to see that operators $\bar{Q}_{1}, \bar{Q}_{0}$ commute with the action of $\partial$. So the action of these operators is well- defined on $\widehat{\Pi}_{0}$.

Comparing definition (4) with hamiltonian (2), we see that evolution (3) is given by the operator

$$
\partial_{\tau}=\bar{Q}_{1}-\bar{Q}_{0}
$$

A local functional $I \in \widehat{\Pi}_{0}$ is called a local integral of motion of the system (3), if $\partial_{\tau} I=0$.The local integrals of motion will be designated as IM. We see from definition (4) that the space of IM of system (3) can be written as:

$$
\widehat{\Pi}_{0} \supset \text { the space of IM of system }(3)=\operatorname{Ker} \bar{Q}_{1} \cap \operatorname{Ker} \bar{Q}_{0} \text {, }
$$

because the operator $\partial_{\tau}$ maps an element from $\widehat{\Pi}_{0}$ to an element from the sum of two different spaces $\widehat{\Pi}_{1} \oplus \widehat{\Pi}_{-1}$.

Introducing translator operator $\mathrm{T}$

$$
T: \Pi_{n} \longrightarrow \Pi_{n+1}, P \otimes e^{n \phi} \longrightarrow P \otimes e^{(n+1) \phi},
$$

where $P \in \Pi_{0}$, define vector fields $Q_{1}, Q_{0}$ on $\Pi_{0}$

$$
\begin{aligned}
& Q_{1}=\bar{Q}_{1} T^{-1} \\
& Q_{0}=\bar{Q}_{0} T
\end{aligned}
$$

Denote the expression $\beta \gamma-\partial \phi$ as $J$. The evolution (3) of the function $J$ is trivial

$$
\partial_{\tau} J(z)=0
$$

Thus we can reduce the space $\Pi_{0}$, imposing the condition

$$
J(z)=0
$$

which is compatible with evolution (3). Reduction (7) of the system (3) gives Maxwell-Bloch equation

$$
\begin{aligned}
& \partial_{\tau} \beta=e^{-\int^{z} d t \beta(t) \gamma(t)}, \\
& \partial_{\tau} \gamma=e^{\int^{z} d t \beta(t) \gamma(t)} .
\end{aligned}
$$


Now we describe Poisson structure of $\beta, \gamma, \partial \phi$ system reduced by the condition $J=0$. Let ue define

$$
\pi_{0}=\Pi_{0} / \mathrm{D} \operatorname{Pol}(J) \Pi_{0}
$$

where D Pol is a set of differential polinomials.

Namely, a differential polinomial $P(\beta, \gamma, \partial \phi)$ from the space $\pi_{0}$ is equivalent to

$$
P(\beta, \gamma, \partial \phi) \sim P(\beta, \gamma, \partial \phi)+\sum_{n=0}^{\infty} R_{n} \partial^{n} J
$$

where $R_{n}$ is differential polinomial in $\beta, \gamma, \partial \phi$. Introduce a Dirac bracket on $\pi_{0}$ $\{,\}^{*}$ with the main property

$$
\{P(\beta, \gamma, \partial \phi), J\}^{*}=0
$$

for any differential polinomial $\mathrm{P}$. Then we have: if $\mathrm{A}, \mathrm{B}$ are differential polinomials in $\beta, \gamma, \partial \phi$, such that $A \sim B$, then $\{\xi, A\}^{*} \sim\{\xi, B\}^{*}$ for arbitrary $\xi$. Using this property and equivalence formula (9) we can treat $\pi_{0}$ as Poisson manifold with coordinates $\beta, \gamma, \partial \beta, \partial \gamma$ etc and Poisson-Dirac bracket:

$$
\begin{aligned}
& \{\gamma(x), \beta(y)\}^{*}=\delta(x-y)+\frac{1}{2} \epsilon(x-y) \gamma(x) \beta(y), \\
& \{\gamma(x), \gamma(y)\}^{*}=-\frac{1}{2} \epsilon(x-y) \gamma(x) \gamma(y), \\
& \{\beta(x), \beta(y)\}^{*}=-\frac{1}{2} \epsilon(x-y) \beta(x) \beta(y),
\end{aligned}
$$

where $\epsilon$ is sign function.

Poisson structure (10) is the first Poisson structure $\{,\}_{1}$ for NLS eq. [7]. After reduction (7) space $\Pi_{n}$ transforms to $\pi_{n}$

$$
\pi_{n}=\pi_{0} \otimes \exp \left(n \int^{z} d \tau \beta \gamma\right)
$$

The action of derivative $\partial$ on $\pi_{n}$ is following

$$
(\partial+n \beta \gamma) \otimes 1
$$

Define $\hat{\pi}_{n}=\pi_{n} / \partial \pi_{n}$ as a space of functionals of the form

$$
\oint d z \operatorname{D} \operatorname{Pol}(\beta, \gamma) e^{n \int^{z} d t \beta \gamma}
$$

After reduction (7) hamiltonian (2) transforms as follows

$$
\oint d z \beta(z) e^{\int^{z} d t \beta(t) \gamma(t)}-\oint d z \gamma(z) e^{-\int^{z} d t \beta(t) \gamma(t)} \in \hat{\pi}_{1} \otimes \hat{\pi}_{-1} .
$$


The action of operators $\bar{Q}_{1}$ and $\bar{Q}_{0}$ is compatible with reduction (7) , i.e. $\bar{Q}_{1} J=\bar{Q}_{0} J=0$. Thus they can be limited from $\Pi_{0}$ to $\pi_{0}$

$$
\begin{aligned}
\bar{Q}_{1}: \pi_{0} \longrightarrow \pi_{1}, \bar{Q}_{1} & =\left\{\cdots, \oint d z \beta e^{\int^{z} d t \beta(t) \gamma(t)}\right\}^{*}, \\
\bar{Q}_{0}: \pi_{0} \longrightarrow \pi_{-1}, & \bar{Q}_{0}=\left\{\cdots, \oint d z \gamma e^{-\int^{z} d t \beta(t) \gamma(t)}\right\}^{*} .
\end{aligned}
$$

The explicit formula for the action (13) of operators $\bar{Q}_{1}$ and $\bar{Q}_{0}$ on the element $P \in \pi_{0}$ is

$$
\begin{aligned}
& \bar{Q}_{1} P=\sum_{n \geq 0} B_{n}^{+} \frac{\partial P}{\partial\left(\partial^{n} \gamma\right)} \otimes e^{\int^{z} d t \beta(t) \gamma(t)}, \\
& \bar{Q}_{0} P=\sum_{n \geq 0} B_{n}^{-} \frac{\partial P}{\partial\left(\partial^{n} \beta\right)} \otimes e^{-\int^{z} d t \beta(t) \gamma(t)},
\end{aligned}
$$

where $P, B_{n}^{ \pm} \in \pi_{0}$ and

$$
\begin{aligned}
\partial_{z}^{n} e^{z} d t \beta(t) \gamma(t) & =B_{n}^{+} e^{\int^{z} d t \beta(t) \gamma(t)}, \\
\partial_{z}^{n} e^{-\int^{z} d t \beta(t) \gamma(t)} & =B_{n}^{-} e^{-\int^{z} d t \beta(t) \gamma(t)} .
\end{aligned}
$$

The MB eq. (8) is treated as Hamilton equation

$$
\begin{aligned}
& \partial_{\tau} \beta=\{\beta, H\}^{*}, \\
& \partial_{\tau} \gamma=\{\gamma, H\}^{*}
\end{aligned}
$$

with brackets $\{,\}^{*}(10)$ and hamiltonian (12).

A local functional $I \in \widehat{\pi}_{0}$ is called a local integral of motion of MB eq., if $\partial_{\tau} I=0$. After imposing the reduction (7) on the formula for evolution (5) we get that the space of IM of MB eq. ${ }^{*}(8)$ is the intersection of kernels of operators $\bar{Q}_{1}$, $\bar{Q}_{0}$

$$
\hat{\pi}_{0} \supset \text { the space of IM of MB eq. }=\operatorname{Ker} \bar{Q}_{1} \cap \operatorname{Ker} \bar{Q}_{0}
$$

Using translator operator $\mathrm{T}$

$$
T: \pi_{n} \longrightarrow \pi_{n+1}, P \otimes e^{n \int^{z} d t \beta \gamma} \longrightarrow P \otimes e^{(n+1) \int^{z} d t \beta \gamma},
$$

where $P \in \pi_{0}$, and explicit formula (14) define vector fields $Q_{1}, Q_{0}$ on $\pi_{0}$

$$
\begin{aligned}
& Q_{1}=\bar{Q}_{1} T^{-1}=\sum_{n \geq 0} B_{n}^{+} \frac{\partial}{\partial\left(\partial^{n} \gamma\right)}, \\
& Q_{0}=\bar{Q}_{0} T=\sum_{n \geq 0} B_{n}^{-} \frac{\partial}{\partial\left(\partial^{n} \beta\right)} .
\end{aligned}
$$


Vector fields $Q_{1}$ and $Q_{0}$ on $\pi_{0}$ satisfy Serre relations for the nilpotent subalgebra $\mathbf{n}_{+}$

$$
a d_{Q_{0}}^{3} \cdot Q_{1}=0 \text { and } a d_{Q_{1}}^{3} \cdot Q_{0}=0
$$

and can be identified with the generators of the nilpotent subalgebra $\mathbf{n}_{+}: e$ and $f \otimes t$.

Thus vector fields $Q_{1}$ and $Q_{0}$ give structure of $\widehat{s l}_{2}$ module to $\pi_{0}$.

Define grading on $\pi_{0}$ :

$$
\begin{aligned}
& \operatorname{deg} \beta=(s=-1, q=1), \operatorname{deg} \gamma=(s=0, q=-1), \\
& \operatorname{deg} \partial=(s=-1, q=0), \quad \operatorname{deg} v_{n}=\left(s=-\frac{1}{2} n(n-1), q=n\right),
\end{aligned}
$$

where q is the isospin with respect to current $J:\left\{J(x), P_{q}(y)\right\}=q \delta(x-y) P_{q}(y)$

Then $\operatorname{deg} \bar{Q}_{1}^{*}=\operatorname{deg} \bar{Q}_{0}^{*}=0$.

\section{Geometrical Picture}

In this section we give geometrical description of the space $\pi_{0}$. Recall that we consider affine algebra $\widehat{s l}_{2}$ without central extension as defined over formal Laurent power series $\mathbf{C}((t)): \widehat{s l}_{2}=s l_{2} \otimes \mathbf{C}((t)) \otimes d$. For $\widehat{s l}_{2}$ we have Cartan decomposition $\widehat{s l} l_{2}=\mathbf{n}_{+} \oplus \mathbf{h} \oplus \mathbf{n}_{-}$, where $\mathbf{n}_{+}$is the nilpotent subalgebra $\mathbf{n}_{+}=\mathbf{C} e \otimes 1 \oplus s l_{2} \otimes \mathbf{C}[[t]]$, $\mathbf{n}_{-}$is the opposite nilpotent subalgebra $\mathbf{n}_{-}=\mathbf{C} f \otimes 1 \oplus s l_{2} \otimes \mathbf{C}\left[t^{-1}\right]$. Let $G$ be the Lie group of the affine algebra $\widehat{s l_{2}}, N_{ \pm}$be the Lie group of the nilpotent subalgebra $\mathbf{n}_{ \pm}, B_{ \pm}$be the Lie group of the Borel subalgebra $\mathbf{b}_{ \pm}=\mathbf{n}_{ \pm} \oplus \mathbf{h}$.

The group $N_{+}$is isomorphic to the big cell $\mathrm{X}$ of the flag manifold $F=$ $B_{-} \backslash G$, which is the orbit of 1 under the action of $N_{+}$. See references [8-10]. The Lie algebra $\widehat{s l}_{2}$ acts infinitesimally from the right by vector fields on $F$ and hence on $N_{+}$. So does the Lie algebra vect $t_{-}=\mathbf{C}\left[t^{-1}\right] t \partial_{t}$, with generators $L_{n}=$ $t^{-n+1} \partial_{t}, n \geq 0$. Denote by $\nu$ the Lie algebra of vector fields on $N_{+}$. It contains two commuting Lie subalgebras: $\mathbf{n}_{+}^{R}$ and $\mathbf{n}_{+}^{L}$ of vector fields of the right and the left infinitesimal action of $\mathbf{n}_{+}$on its Lie group. The vector field of the left infinitesimal action of $\beta \in \mathbf{n}_{+}$on $N_{+}$denoted by $\beta^{L}$. The Lie algebra $\mathbf{n}_{+}^{R}$ is a part of a larger subalgebra of $\nu$, which is isomorphic to $\tilde{\mathbf{g}}=\widehat{s l}_{2} \times$ vect $t_{-}$. The vector field of the right infinitesimal action of $\alpha \in \tilde{\mathbf{g}}$ on $N_{+}$will be denoted by $\alpha^{R}$.

For $j \in \mathbf{C}$ let $M_{j}$ be Verma module over $\widehat{s l}_{2}$ of the $s l_{2}$ spin $j$ with the highest vector $v_{j}$

$$
\mathbf{n}_{+} \cdot v_{j}=0, \quad h \cdot v_{j}=j v_{j}, \quad M_{j}=U\left(\mathbf{n}_{-}\right) \cdot v_{j} .
$$

$M_{j}^{*}$ is the module contragradient to $M_{j}$ with pairing $<,>: M_{j}^{*} \times M_{j} \rightarrow \mathbf{C}$. Following [2] we describe a geometrical construction of $M_{j}^{*}$. Let $\omega$ be Cartan anti-involution on $\widehat{s l}_{2}$, mapping $e, f \otimes t$ to $f, e \otimes t^{-1}$.

Define the right action of $y \in \widehat{s l}_{2}$ on $x \in M_{j}^{*}$ as follows:

$$
<x \cdot y, z>=<x, \omega(y) \cdot z>, \quad z \in M_{j}
$$


The module $M_{j}^{*}$ can be identified with space of functions $\mathbf{C}(X)$ on the big cell $\mathrm{X}$ with respect to a twisted action. The right action of $\beta \in \widehat{s l}_{2}$ on $M_{j}^{*}$ gives under this identification the action of

$$
\beta^{R}+j F(\beta) \text { on } \mathbf{C}(X),
$$

where $F(\beta)$ is function on X. We have $F(h)=1$ and $F(\beta)=0$ for $\beta \in \mathbf{n}_{+}$.

Let vector $v_{m}$ be singular vector of Verma module $M_{j}, v_{m}=P \cdot v_{j}$ for some element $P \in U\left(\mathbf{n}_{-}\right)$and $R \cdot v_{m}=0$ for any element $R \in U\left(\mathbf{n}_{+}\right)$. This singular vector defines homomorphism of $\widehat{s l}_{2}$-modules

$$
i_{P}: M_{m} \rightarrow M_{j}, \quad u \cdot v_{m} \rightarrow(u P) \cdot v_{j}
$$

for any $u \in U\left(\mathbf{n}_{-}\right)$. The map $i_{P}$ commutes with the $\widehat{s l}_{2}$-action and is called intertwining opertor.

The left action of the element $\beta \in \mathbf{n}_{+}$on $x \in M_{j}^{*}$ can be defined as follows

$$
<\beta \cdot x, u \cdot v_{j}>=<x,(u \omega(\beta)) \cdot v_{j}>, u \in U\left(\mathbf{n}_{-}\right)
$$

Let $\bar{P}$ be the image of $P \in U\left(\mathbf{n}_{-}\right)$under isomorphism $U\left(\mathbf{n}_{-}\right) \rightarrow U\left(\mathbf{n}_{+}\right)$, which maps generators $e, f \otimes t$ to $f, e \otimes t^{-1}$. The homomorphism $\mathbf{n}_{+} \rightarrow \nu$, mapping $\alpha \in \mathbf{n}_{+}$to $\alpha^{L}$, can be extended uniquly to homomorphism from $U\left(\mathbf{n}_{+}\right)$to the algebra of differential operators on X . Let $u^{L}$ be image of $u \in U\left(\mathbf{n}_{+}\right)$.

It is known [2] that homomorphism $i_{P}^{*}: M_{j}^{*} \rightarrow M_{m}^{*}$ dual to (17) can be realised as differential operator $\bar{P}^{L}$ on X. For example, consider the map $i_{f}$ : $M_{-2} \rightarrow M_{0}, u \cdot v_{-2} \rightarrow(u f) \cdot v_{0}$. Then dual map $i_{f}^{*}: M_{0}^{*} \rightarrow M_{-2}^{*}$ is given by the left infinitesimal action $e^{L}$ on $\mathbf{C}(X)$ treated as $M_{0}^{*}$.

Let us study further the left action of $U\left(\mathbf{n}_{-}\right)$on $\mathbf{C}(X)$. In order to simplify formulas introduce the Chevalle basis of $\mathbf{n}_{+}: e_{1}=e, e_{0}=f \otimes t$. For $F(\beta), \beta \in \widehat{s l}_{2}$ we have following

$$
\left[e_{i}^{L}, \beta^{R}\right]=2(-)^{i} F(\beta) e_{i}^{L}, \quad i=0,1 .
$$

For proving (18) it's sufficient to consider $e_{1}^{L}$ as $\widehat{s l}_{2}$-homomorphism from $M_{0}^{*}$ to $M_{-2}$ and $e_{0}^{L}$ as $\widehat{s l}_{2}$-homomorphism from $M_{0}^{*}$ to $M_{2}$. Here we treat $M_{j}^{*}$ as $\mathbf{C}(X)$ with twisted action. After identifing $\mathbf{C}\left(N_{+}\right)$with $\left(U\left(\mathbf{n}_{+}\right)\right)^{*}$ introduce grading on $\mathbf{C}(X) \simeq \mathbf{C}\left(N_{+}\right)$with respect to degree of $t$ and action of $\mathbf{h}$

$$
\operatorname{deg} e \otimes t^{n}=(n, 1), \operatorname{deg} f \otimes t^{n}=(n,-1), \operatorname{deg} h \otimes t^{n}=(n, 0)
$$

We prove that the space of functions $\mathbf{C}\left(N_{+} / H_{+}\right)$on the homogeneous space $N_{+} / H_{+}$is isomorphic to $\pi_{0}$ as $\mathbf{n}_{+}$-modules. Indeed, vector fields $Q_{1}, Q_{0}$ (15) define structure of $\mathbf{n}_{+}$-module on $\pi_{0}$. Let $x_{i}, y_{i}$ be coordinates on $\pi_{0}$

$$
x_{i+1}=\partial^{i} \beta, \quad y_{i}=\partial^{i} \gamma, \quad i \geq 0
$$


Then for the vector fields $Q_{1}, Q_{0}$ on $\pi_{0}$ we have (see (15))

$$
\begin{aligned}
Q_{1} & =\frac{\partial}{\partial y_{0}}+x_{1} y_{0} \frac{\partial}{\partial y_{1}}+\left(x_{1}^{2} y_{0}^{2}+x_{2} y_{0}+x_{1} y_{1}\right) \frac{\partial}{\partial y_{2}}+\cdots \\
Q_{0} & =\frac{\partial}{\partial x_{1}}-x_{1} y_{0} \frac{\partial}{\partial x_{2}}+\left(x_{1}^{2} y_{0}^{2}-x_{2} y_{0}+x_{1} y_{1}\right) \frac{\partial}{\partial x_{3}}+\cdots
\end{aligned}
$$

Let $\mathrm{X}$ be an operator on $\pi_{0}$ of the form $\sum_{i}\left(X_{i} \partial / \partial x_{i}+Y_{i} \partial / \partial y_{i}\right)$, then define its shift term as all the terms $X_{i} \partial / \partial x_{i}$ or $Y_{i} \partial / \partial y_{i}$, for which $X_{i}$ or $Y_{i}$ is constant. From (20) shift terms of $Q_{1}$ and $Q_{0}$ are $\partial / \partial y_{0}$ and $\partial / \partial x_{1}$. The shift term of $\left[Q_{1}, Q_{0}\right]$ is $0 . Q_{1}$ and $Q_{0}$ satisfy Serre relation and generate $\mathbf{n}_{+}$. In our notation we can treat $Q_{1}$ as $e$ and $Q_{0}$ as $f \otimes t$. It's easy to see that vector fields corresponding to subalgebra $\mathbf{h}_{+}$have no shift terms, and shift term of $e \otimes t^{n}$ is $\partial / \partial y_{n}, \quad n \geq 0$, while shift term of $f \otimes t^{n}$ is $\partial / \partial x_{n}, \quad n \geq 1$.

Consider the module $\pi_{0}^{*}$ over $\mathbf{n}_{+}$, dual to $\pi_{0}$. We can identify $\pi_{0}$ with $\pi_{0}^{*}$ as linear spaces, choosing the monomials $x_{k_{1}} / k_{1} ! \cdots x_{k_{n}} / k_{n}$ ! and $y_{k_{1}} / k_{1} ! \cdots y_{k_{n}} / k_{n}$ ! as an ortonormal basis. The formulas for the action of $\mathbf{n}_{+}$on $\pi_{0}^{*}$ are obtained from the formulas for its action on $\pi_{0}$ by interchainging $x_{n}\left(y_{n}\right)$ and $\partial / \partial x_{n}\left(\operatorname{resp} . \partial / \partial y_{n}\right)$. Combinations of $Q_{0}, Q_{1}$, corresponding to $\mathbf{h}_{+}$, act on $1^{*} \in \pi_{0}^{*}$ by 0 (because they have not shift terms).

Let us introduce

$$
N=U\left(\mathbf{n}_{+}\right) \otimes_{U\left(\mathbf{h}_{+}\right)} \mathbf{C},
$$

$\mathbf{n}_{+}$-module, induced from the trivial one-dimentional representation of subalgebra $\mathbf{h}_{+}$. Since action of $\mathbf{h}_{+}$on $1 \otimes 1 \in N$ is trivial, there is unique $\mathbf{n}_{+}$-homomorphism $N \rightarrow \pi_{0}$, sending $1 \otimes 1 \in N$ to $1^{*} \in \pi_{0}^{*}$, and $\left(e \otimes t^{n_{1}}\right) \cdots\left(e \otimes t^{n_{k}}\right)\left(f \otimes t^{m_{1}}\right) \cdots(f \otimes$ $\left.t^{m_{l}}\right) \otimes 1$ maps to $y_{n_{1}} \cdots y_{n_{k}} x_{m_{1}} \cdots x_{m_{k}} \cdot 1^{*}+$ lower order terms. Therefore map $N \rightarrow \pi_{0}$ has no kernel. $N$ and $\pi_{0}$ coinside as linear spaces with respect to graiding (16) and (19). Thus

$$
\pi_{0} \simeq\left(U\left(\mathbf{n}_{+}\right) \otimes_{U\left(\mathbf{h}_{+}\right)} \mathbf{C}\right)^{*} \simeq \mathbf{C}\left(N_{+} / H_{+}\right)
$$

as $\mathbf{n}_{+}$-modules.

Recall that $\mathbf{h}_{ \pm}$is commuting subalgebra of $\mathbf{n}_{ \pm}$, generated by $h_{ \pm i}=h \otimes$ $t^{ \pm i}, \quad i=1,2, \cdots$. Consider eq. (18) for $\beta=h_{-1}$

$$
\left[e_{i}^{L}, h_{-1}^{R}\right]=(-)^{i} 2 F\left(h_{-1}\right) e_{i}^{L}, \quad i=0,1
$$

as vector fields on $\mathbf{C}\left(N_{+}\right)$. For the function $F\left(h_{-1}\right)$ on the group $N_{+}$we have

$$
x_{+}^{R} \cdot F\left(h_{-1}\right)=0,
$$

for $x_{+} \in \mathbf{h}_{+}$. This can be derived from commutator (22). Indeed,

$$
\left[x_{+}^{R},\left[e_{i}^{L}, h_{-1}\right]\right]=-\left[e_{i}^{L},\left[h_{1}^{R}, x_{+}^{R}\right]\right]-\left[h_{-1}^{R},\left[x_{+}^{R}, e_{i}^{L}\right]\right]=0 .
$$


So for $i=0,1\left(x_{+}^{R} \cdot F\left(h_{-1}\right)\right) \cdot e_{i}^{L}=0$ and then (23). Eq. (23) gives us example of $\mathbf{h}_{+}^{R}$ - invariant functions on $\mathbf{C}(X)$. It's obvious that the right action of $\mathbf{h}_{-}$on $F\left(h_{-1}\right)$ preserves $\mathbf{h}_{+}$-invariancy, thus $\beta^{R} \cdot F\left(h_{-1}\right)$ for $\beta \in \mathbf{h}_{-}$is $\mathbf{h}_{+}$-invariant. Other families of $\mathbf{h}_{+}$invariant functions on $\mathbf{C}(X)$ are given by $F(f), F\left(e \otimes t^{-1}\right)$ and the right action of $\mathbf{h}_{-}$on them.

Thus we can treat $(22)$ as vector fields on $\mathbf{C}\left(N_{+} / H_{+}\right)$, because $e_{i}^{L}, h_{-1}^{R}$ commutes with $\mathbf{h}_{+}^{R}$, and $F\left(h_{-1}\right)$ is $\mathbf{h}_{+}$-invariant.

Using isomorphism (21) we can identify $e_{i}^{L}$ acting on $\mathbf{C}\left(N_{+} / H_{+}\right)$with $Q_{i}$ acting on $\pi_{0}$. The element $F\left(h_{-1}\right)$ of $\mathbf{C}\left(N_{+} / H_{+}\right)$has grading $(16): \operatorname{deg} F\left(h_{-1}\right)=$ $(-1 ; 0)$. The only image of $F\left(h_{-1}\right)$ in $\pi_{0}$ under isomorphism $\mathbf{C}\left(N_{+} / H_{+}\right) \rightarrow \pi_{0}$ of the same degree is const $\cdot x_{1} y_{0}(=$ const $\cdot \beta \gamma)$, degx $x_{1} y_{0}=(-1 ; 0)$.

So using (22) and isomorphism (21), we have

$$
\left[Q_{i}, \eta_{-1}\right]=\text { const } \cdot(-)^{i} 2 x_{1} y_{0} Q_{i}, \quad i=0,1
$$

as vector fields on $\pi_{0}$, where $\eta_{-1}$ is unknown vector field on $\pi_{0}$.

We prove that the vector field $\eta_{-1}$ is proportional to the vector field of derivative $\partial=\sum x_{i} \partial / \partial x_{i-1}+y_{i} \partial / \partial y_{i-1}$. If we choose const $=-\frac{1}{2}$ then

$$
\eta_{-1}=\partial
$$

on $\pi_{0}$. Indeed, operators $\bar{Q}_{1}=T Q_{1}$ and $\bar{Q}_{0}=T^{-1} Q_{0}$ commute the action of $\partial$ (11) on $\pi_{0}$. That is why

$$
\left[Q_{1}, \partial\right]=T^{-1}[\partial, T] Q_{1}=x_{0} y_{1} Q_{1} \text { and }\left[Q_{0}, \partial\right]=T\left[\partial, T^{-1}\right]=-x_{0} y_{1} Q_{0}
$$

So the image of the action of $h_{-1}^{R}$ on $\mathbf{C}\left(N_{+} / H_{+}\right)$under isomorphism (21) is the action of $\partial$ on $\pi_{0}$.

\section{Cohomology Computation}

In this section we describe the space of IM of MB eq. using a double complex [2]. Let $B^{*}\left(\widehat{s l}_{2}\right)$ be the dual of BGG resolution for $\widehat{s l}_{2}[11]$

$$
B^{*}\left(\widehat{s l}_{2}\right)=\bigoplus_{j \geq O} B^{j}\left(\widehat{s l}_{2}\right)
$$

where $B^{0}\left(\widehat{s l}_{2}\right)=M_{0}^{*}$ and $B^{j}\left(\widehat{s l}_{2}\right)=M_{2 j}^{*} \oplus M_{-2 j}^{*}$. Here $M_{j}^{*}$ is contragradient module to the Verma one with $s l_{2}$ spin $j$ and level $\mathrm{k}=0$.

The Verma module $M_{0}$ contains singular vectors, labeled by the elements of the Weyl group of $\widehat{s l}_{2}$. Denote this vectors by $w_{0}$ and $w_{ \pm j}, j=1,2, \cdots$. The weights of $w_{ \pm j}$ are $\pm 2 j$. The action of $U\left(\mathbf{n}_{-}\right)$on $w_{ \pm j}$ generates the submodule $M_{w_{ \pm j}}$ isomorphic to $M_{ \pm 2 j}$. Let $l\left(w_{ \pm j}\right)=j$. Choose two elements $w, w^{\prime}$ of the Weyl group, such that $l(w)=l\left(w^{\prime}\right)+1$. Then $\bar{P}_{w, w^{\prime}}^{L}$ is a map, dual to embeding (17) $i_{w, w^{\prime}}: M_{w} \rightarrow M_{w^{\prime}}$. (We identify $M_{j}^{*}$ with $\left.\mathbf{C}(X)\right)$. 
The 0-th cohomology of $B^{*}\left(\widehat{s l}_{2}\right)$ is one-dimentional and all higher ones vanish. Differentials $\delta^{j}: B^{j}\left(\widehat{s l}_{2}\right) \rightarrow B^{j+1}\left(\widehat{s l}_{2}\right)$ of $B^{*}\left(\widehat{s l}_{2}\right)$ can be written in a common way:

$$
\delta^{j}=\sum_{l(w)=j, l\left(w^{\prime}\right)=j+1} \epsilon_{w, w^{\prime}} \bar{P}_{w, w^{\prime}}^{L} .
$$

From this formula the first differential $\delta^{1}$, mapping $M_{0}^{*} \rightarrow M_{2}^{*} \oplus M_{-2}^{*}$ is $e_{1}^{L}-e_{0}^{L}$ as the action on $\mathbf{C}(X)$.

The right action of $\widehat{s l}_{2}$ on complex $B^{*}\left(\widehat{s l}_{2}\right)$ commutes with the differentials $\delta^{j}$. This valid for $\mathbf{h}_{+} \subset \widehat{s l_{2}}$. So we can take quotient of $B^{*}\left(\widehat{s l}_{2}\right)$ by the right action of Lie subgroup $H_{+}$of $N_{+}$. Denote this complex by $F^{*}\left(\widehat{s l}_{2}\right)$ :

$$
F^{*}\left(\widehat{s l}_{2}\right)=\bigoplus_{j \geq 0} F^{j}\left(\widehat{s l}_{2}\right),
$$

where $F^{0}\left(\widehat{s l}_{2}\right)=\pi^{(0)}$ and $F^{j}\left(\widehat{s l}_{2}\right)=\pi^{(2 j)} \oplus \pi^{(-2 j)}$, here $\pi^{( \pm 2 j)}$ denotes space of $\mathbf{h}_{+}$-invariants of $M_{ \pm 2 j}^{*}$.

The right action of $\mathbf{h}_{-}$on $B^{*}\left(\widehat{s l}_{2}\right)$ gives rise to $\mathbf{h}_{-}$-action on $F^{*}\left(\widehat{s l}_{2}\right)$, because for $x \in \mathbf{h}_{-}$function $F(x)$ is $\mathbf{h}_{+}$-invariant . The action of $x \in \mathbf{h}_{-}$on $\pi^{(j)}$ is given by

$$
x^{R}+j F(x)
$$

This action commutes with the differentials of the complex $F^{*}\left(\widehat{s l}_{2}\right)$, that is why the action of $\mathbf{h}_{-}$is defined on the cohomologies of $F^{*}\left(\widehat{s l}_{2}\right)$.

For description of the space of IM of MB eq. cohomologies of $F^{*}\left(\widehat{s l}_{2}\right)$ should be computed. We prove that cohomologies of $F^{*}\left(\widehat{s l}_{2}\right)$ are isomorphic to $\wedge^{*}\left(\mathbf{h}_{+}^{*}\right)$.

Since $B^{*}\left(\widehat{s l}_{2}\right)$ is injective resolution of the trivial representation of $\mathbf{n}_{+}$, the cohomologies of $F^{*}\left(\widehat{s l}_{2}\right)$ coincide with the cohomologies $H^{*}\left(\mathbf{n}_{+}, \pi_{0}\right)$ of the Lie algebra $\mathbf{n}_{+}$with coefficients in the module $\pi_{0}$ [1]. Because $\pi_{0}$ can be identified with $\left(U\left(\mathbf{n}_{+}\right) \otimes_{U\left(\mathbf{h}_{+}\right)} \mathbf{C}\right)^{*}$, we have by Shapiro lemma:

$$
H^{*}\left(\mathbf{n}_{+}, \pi_{0}\right) \simeq H^{*}\left(\mathbf{h}_{+}, \mathbf{C}\right) \simeq \wedge^{*}\left(\mathbf{h}_{+}^{*}\right)
$$

In [2] it was proved that the action of $\mathbf{h}_{-}$on $H^{*}\left(\mathbf{n}_{+}, \pi_{0}\right)$ is trivial. In particular, the operator $h_{-1}$ acts trivially on the cohomologies. We already know that its action on $\pi_{0}$ consides with the action of $\partial$. Consider now the action of $h_{-1}$ on $\pi^{( \pm 2 j)}$. It is given by $h_{-1}^{R} \pm 2 j F\left(h_{-1}\right)$. The image of $F\left(h_{-1}\right)$ under isomorphism $\mathbf{C}\left(N_{+} / H_{+}\right) \rightarrow \pi_{0}$ is equal to $-\frac{1}{2} \beta \gamma$. So the action of $h_{-1}$ on $\pi^{( \pm 2 j)}$ coincides with the action (11) of derivative $\partial$ on $\pi_{\mp j}$ and we have isomorphism $\pi^{( \pm 2 j)}=\pi_{\mp j}$ with respect to the action of $\mathbf{n}_{+}$. Thus $F^{0}\left(\widehat{s l}_{2}\right)=\pi_{0}, F^{j}\left(\widehat{s l}_{2}\right)=\pi_{-j} \oplus \pi_{j}$ and the first differential $\delta^{1}: \pi_{0} \rightarrow \pi_{-1} \oplus \pi_{1}$ is equal to $\delta^{1}=\bar{Q}_{1}-\bar{Q}_{0}$. We see that differential $\delta^{1}$ coincides with the action of MB hamiltinian on $\pi_{0}$. This observation enables 
us to compute the space of IM of MB eq. Indeed, the space of IM is isomorphic to the kernel of the map $\delta^{1}=\bar{Q}_{1}^{*}-\bar{Q}_{0}^{*}: \hat{\pi}_{0} \rightarrow \hat{\pi}_{-1} \oplus \hat{\pi}_{1}$. Recall that $\hat{\pi}_{n}=\pi_{n} / \partial \pi_{n}$. In order to use the complex $F^{*}\left(\widehat{s l}_{2}\right)$ for computing IM we should get rid of total derivatives in $z$, i.e. consider $F^{*}\left(\widehat{s l}_{2}\right) / \partial F^{*}\left(\widehat{s l}_{2}\right)$. For these reasons a double complex is used.

Now the main result can be formulated : the space of integrals of motion of the MB eq. (8) is lineary spanned by elements $H_{m}, m=1,2, \cdots$, where $\operatorname{deg} H_{m}=$ $(-m, 0)$.

Since $\partial=h_{-1}$ commutes with the differentials of $F^{*}\left(\widehat{s l}_{2}\right)$, we can consider the doube complex:

$$
\mathbf{C} \longrightarrow F^{*}\left(\widehat{s l}_{2}\right) \stackrel{ \pm h_{-1}}{\longrightarrow} F^{*}\left(\widehat{s l}_{2}\right) \longrightarrow \mathbf{C}
$$

Using the spectral sequence, in which $h_{-1}$ is the 0 th differential, one gets that the 1st cohomology of the double complex $H_{t o t}^{1}$ is isomorphic to the space of IM. We can also compute this cohomology using the other spectral sequence. Because $h_{-1}$ acts trivially on $H^{1}\left(F^{*}\left(\widehat{s l}_{2}\right)\right), H_{\text {tot }}^{1} \simeq H^{1}\left(F^{*}\left(\widehat{s l}_{2}\right)\right) \simeq \mathbf{h}_{+}^{*}$. Therefore space of IM is linearly spanned by elements $H_{m}$ of degree $(\mathrm{s}=-\mathrm{m}, \mathrm{q}=0)$ for $\mathrm{m}=1,2 \cdots$.

\section{Construction of IM and vector fields}

Now we show explicitly how IM are connected with the first cohomology class $H^{1}\left(F^{*}\left(\widehat{s l}_{2}\right)\right)$. Consider the element $\mathcal{H}$ of cohomology class $H^{1}\left(F^{*}\left(\widehat{s l}_{2}\right)\right) \subset \pi_{-1} \oplus \pi_{1}$. If we apply $\partial$ to $\mathcal{H}$, we obtain a trivial cycle. So there exists element $h \in \pi_{0}$ such that

$$
\delta^{1} \cdot h=\partial \mathcal{H}
$$

where $\delta^{1}$ is the first differential of the complex $F^{*}\left(\widehat{s l}_{2}\right)$ equal to the MB flow: $\delta^{1}=\bar{Q}_{1}-\bar{Q}_{0}=\partial_{\tau}$. By construction $h$ is not total derivative, so it belongs to IM. Thus, formula (29) explicitly gives isomorphism between the spase of IM and the cohomology class $H^{1}\left(F^{*}\left(\widehat{s l}_{2}\right)\right)$.

For example, consider two lowest IM: $h^{(-2)}=r q \in \pi_{0}$ and $h^{(-3)}=r \partial q \in \pi_{0}$ of degrees $(-2 ; 0)$ and $(-3 ; 0)$. They are connected via formula $(29)$ with $j^{(-1)}=$ $\frac{1}{4} \gamma e^{-\int^{z} d t \beta(t) \gamma(t)} \in H^{1}\left(F^{*}\left(\widehat{s l}_{2}\right)\right)$ and $j^{(-2)}=\frac{1}{4} \beta \gamma^{2} e^{-\int^{z} d t \beta(t) \gamma(t)} \in H^{1}\left(F^{*}\left(\widehat{s l}_{2}\right)\right)$ of degrees $(-1 ; 0)$ and $(-2 ; 0)$.

Denote by $H_{m} \in \hat{\pi}_{0}$ element of the space of IM of MB eq. of degree $(-\mathrm{m} ; 0)$. Let $\eta_{m}$ be the vector field on $\pi_{0}$ :

$$
\eta_{m}=\left\{\cdots, H_{m}\right\}^{*}
$$

It is easy to see that $\eta_{1}=\partial$. We can treat $\eta_{m}$ as vector fields on $N_{+} / H_{+}$under isomorphism $\pi_{0} \simeq \mathbf{C}\left(N_{+} / H_{+}\right)$. On the other hand the right action of generator $h_{-m}, \quad m=1,2, \cdots$ of the subalgebra $\mathbf{h}_{-} \in \widehat{s l}_{2}$ on $N_{+} / H_{+}$also defines vector 
fields $\mu_{m}$ on $N_{+} / H_{+}$. Now we formulate the result : the vector fields $\mu_{m}$ coincide with $\eta_{m}, m=1,2 \cdots$.

The statement was already proved for $m=1$. We have shown that action of $h_{-1}$ on $\pi_{0}$ gives us $\partial$. So does vector field $\eta_{1}=\left\{\cdots, H_{1}\right\}^{*}$, where $H_{1}=\oint d z r q$. Consider now commutator of operators $\bar{Q}_{1}$ and $\eta_{m}$, acting on $P \in \pi_{0}$

$$
\left[\bar{Q}_{1}, \eta_{m}\right] P=\left\{\left\{\oint d z \beta e^{\int^{z} d t \beta(t) \gamma(t)}, H_{m}\right\}^{*}, P\right\}^{*}=0
$$

since $\left\{\oint d z \beta e^{\int^{z} d t \beta(t) \gamma(t)}, H_{m}\right\}^{*}=0$ by the definition of IM. The same is valid for $\bar{Q}_{0}$.

Now we can compute commutator of vector fields $Q_{i}$ and $\eta_{m}$ on $\pi_{0}$. Using expressions for $\bar{Q}_{1}=T Q_{1}$ and $\bar{Q}_{0}=T^{-1} Q_{0}$ one can derive:

$$
\left[Q_{i}, \eta_{m}\right]=-f_{m}^{i} Q_{i}
$$

as vector fields on $\pi_{0}$, where $f_{m}^{i}$ - certain function on $\pi_{0}$.

Let us consider now eq.(18) on $\mathbf{C}\left(N_{+}\right)$and vector fields $\beta$ on $\mathbf{C}\left(N_{+}\right)$, satisfing it. It is obvious that, if vector fields $\alpha$ and $\beta$ satisfy (18), then $[\alpha, \beta]$ satisfies it too. So we have Lie algebra of vector fields on $\mathbf{C}\left(N_{+}\right)$, satisfing (18). In [2] it was shown, that this Lie algebra is isomorphic to $\tilde{\mathrm{g}}$. The only vector fields, satisfing (18), which in addition $\mathbf{h}_{+}$-invariant, are generated by right action of $\mathbf{h}_{-}$on $\mathbf{C}\left(N_{+}\right)$, i.e. $\mu_{m}$. On the other hand vector fields $\eta_{m}$ can be lifted to $\mathbf{h}_{+}$-invariant vector fields $\tilde{\eta}_{m}$ on $N_{+}$, which satisfy relation (31) and thus (18). Comparing the degrees of vector fields $\tilde{\eta}_{m}$ and $\mu_{m}$ we get the statement.

Now proof of well- known result [7]: flows generated by IM of MB eq. (i.e. the positive AKNS hamiltonians) commutes with each other $\left\{H_{m}, H_{n}\right\}=0$ for $m, n=1,2, \cdots$ is obvious.

Because of commutativity of subalgebra $\mathbf{h}_{-}$the vector fields $\mu_{m}$, generating by the right action of $h_{-m} \in \mathbf{h}_{-}$commute with each other. So do corresponding vector fields $\eta_{m}$ on $\pi_{0}$. Using definition of $\eta_{m}$ (30), we find commutativity of the positive AKNS hamiltonians.

\section{Concluding Remarks}

In this paper we showed the relation between classical Maxwell-Bloch equation and AKNS hierarchy with the geometry of the affine Lie group coset $N_{+} / H_{+}$. The simplisity of the action of the Maxwell-Bloch hamiltonian and the integrals of motion on the phase space enables to treate coordinates on the coset $N_{+} / H_{+}$as "scatering data". The technique of the Lie group interpretation of the phase space was transmited from classical Toda theories [1-2] to the classical Maxwell-Bloch equation. It is possible to give natural Poisson group structure on the MaxwellBloch phase space contrary to the Toda phase space. Namely, the action of the hamiltonian on the MB phase space can be understood as a Poisson action of a 
Poisson-Lie group on a Poisson manifold. Quantization of such Poisson structures leads to a quantum version of Maxwell-Bloch equation. Another way to quantize Maxwell-Bloch equation is to use vertex operator algebra methods as it was done for Toda theories [1].

The lattice version of MB eq. can be given as follows. Consider functions

$$
X(z)=\beta(z) e^{\phi(z)} \text { and } Y(z)=\gamma(z) e^{-\phi(z)}
$$

and introduce $X_{i}=X(i a), Y_{i}=Y(i a), \quad i \in \mathbf{Z}$ as lattice variables, where $a$ is a lattice parameter. The Poisson bracket of $X_{i}$ and $Y_{j}$ computed using definition (1) is a classical limit of a q- commutator of lattice variables $\left[X_{i}, Y_{j}\right]_{q}=\delta_{i j}$. We will describe the lattice version of $\mathrm{MB}$ eq. in the forthcoming paper.

Such kind of latticization was proposed by one of the authors (B. F) [12] and studied for Toda systems in $[13,14]$.

\section{Acknoledgements}

We are grateful to A. Belavin and members of his Seminar in Landau Institute: A. Kadeishvili, S. Kryukov, M. Lashkevich, S. Parkhomenko, V. Postnikov and Ya. Pugai for useful discussions.

\section{References}

1. B. Feigin, E. Frenkel, in Proceedings of the Summer School Integrable Systems and Quantum Groups, Montecatini Terme, Italy, June 1993, Lect. Notes in Math., Springer- Verlag.

2. B. Feigin, E. Frenkel, Generalized KdV Flows and Nilpotent Subgroups of Affine Kac-Moody Groups, hep-th 9311171

3. M. Ablovitz, D. Kaup, A. Newell, H. Segur, Stud. Appl. Math 53 (1974) $249-315$

4. A. Mikhailov, M. Olshanetsky, V. Perelomov, Commun. Math. Phys. 79 (1981) 473-488

5. I. Gabitov, V. Zakharov, A. Mikhailov Teor. Mat. Fiz. (in Russian) 63 (1985) , 11-31

6. A. Leznov, V. Man'ko, S. Chumakov Teor. Mat. Fiz. (in Russian) 63 (1985) , 50-63

7. L. Faddeev, L. Takhtajan, Hamiltonian methods in the Theory of Solitons, Springer- Verlag, 1984

8. D. Kazhdan, G. Lusztig, Proc. Symp. in Pure Math. 36 (1980), 185-203

9. V. Kac, D. Peterson, Proc. Natl. Acad. Sci. USA 80 (1983), 1778-1782

10. A. Pressley, G. Segal, Loop Groups, Clarendon Press, Oxford 1986

11. J. Bernstein, I. Gelfand, S. Gelfand, in Representation of Lie groups, ed. I. Gelfand, 21-64, Wiley, New York 1975

13. B. Feigin, Lectures in Landau Institute for Theoretical Physics, Moscow (1992) 
13. S. Kryukov, Ya. Pugay, Lattice W-algebras and quantum groups, hep-th 9310154

14. B. Enriquez, B. Feigin, Integrals of Motion of Classical Lattice Sin-Gordon System, hep-th 9409075 